\title{
Formulation Predictive Dissolution (fPD) Testing to Advance Oral Drug Product Development: an Introduction to the US FDA Funded ' $21^{\text {st }}$ Century BA/BE' Project
}

Bart Hens ${ }^{1,2}$, Patrick Sinko ${ }^{1}$, Nicholas Job ${ }^{1}$, Meagan Dean ${ }^{1}$, Jozef Al-Gousous ${ }^{1}$, Niloufar Salehi ${ }^{3}$, Robert M. Ziff ${ }^{3}$, Yasuhiro Tsume ${ }^{1}$, Marival Bermejo ${ }^{1,4}$, Paulo Paixão ${ }^{1,5}$, James G. Brasseur ${ }^{6,7}$, Alex Yu ${ }^{1}$, Arjang Talattof ${ }^{1}$, Gail Benninghoff ${ }^{1}$, Peter Langguth ${ }^{8}$, Hans Lennernäs ${ }^{9}$, William L. Hasler ${ }^{10}$, Luca Marciani ${ }^{11}$, Joseph Dickens ${ }^{12}$, Kerby Shedden ${ }^{12}$, Duxin Sun ${ }^{1}$, Gregory E. Amidon ${ }^{1}$, Gordon L. Amidon ${ }^{1 *}$

${ }^{1}$ Department of Pharmaceutical Sciences, College of Pharmacy, University of Michigan, Ann Arbor, Michigan 48109-1065, USA

${ }^{2}$ Department of Pharmaceutical and Pharmacological Sciences, KU Leuven, Leuven, 3000, Leuven, Belgium

${ }^{3}$ Center for the Study of Complex Systems and Department of Chemical Engineering, University of Michigan, Ann Arbor, Michigan 48109-2136, USA

${ }^{4}$ Department Engineering Pharmacy Section, Miguel Hernandez University, San Juan de Alicante, 03550 Alicante, Spain

${ }^{5}$ Research Institute for Medicines (iMed.ULisboa), Faculty of Pharmacy, Universidade de Lisboa, Av. Professor Gama Pinto, 1649-003 Lisboa, Portugal

${ }^{6}$ Department of Mechanical and Nuclear Engineering, Pennsylvania State University, University Park, PA 16802, USA

${ }^{7}$ Department of Aerospace Engineering Sciences, University of Colorado Boulder, Boulder, CO 80309, USA

${ }^{8}$ Department of Pharmaceutical Technology and Biopharmaceutics, Johannes Gutenberg University Mainz, Staudinger Weg 5, Mainz D-55099, Germany

${ }^{9}$ Department of Pharmacy, Uppsala University, Uppsala, Sweden

${ }^{10}$ Department of Internal Medicine, Division of Gastroenterology, University of Michigan, Ann Arbor, Michigan 48109, USA

${ }^{11}$ Nottingham Digestive Diseases Centre and 2NIHR Nottingham Biomedical Research Centre at Nottingham University Hospitals NHS Trust and the University of Nottingham, Nottingham NG7 2UH, United Kingdom

${ }^{12}$ Department of Statistics, University of Michigan, Ann Arbor, Michigan 48109, United States

\section{Corresponding Author}

* Dr. Gordon L. Amidon

428 Church St.

College of Pharmacy

University of Michigan

Ann Arbor, MI 48109-1065

Phone: +(1) 734-764-2226 
Fax: $\quad+(1) 734-764-6282$

Email: glamidon@med.umich.edu 
Graphical abstract

A New Era for Biorelevant Formulation Predictive Dissolution Testing: $21^{\text {st }}$ Century BE/BA studies
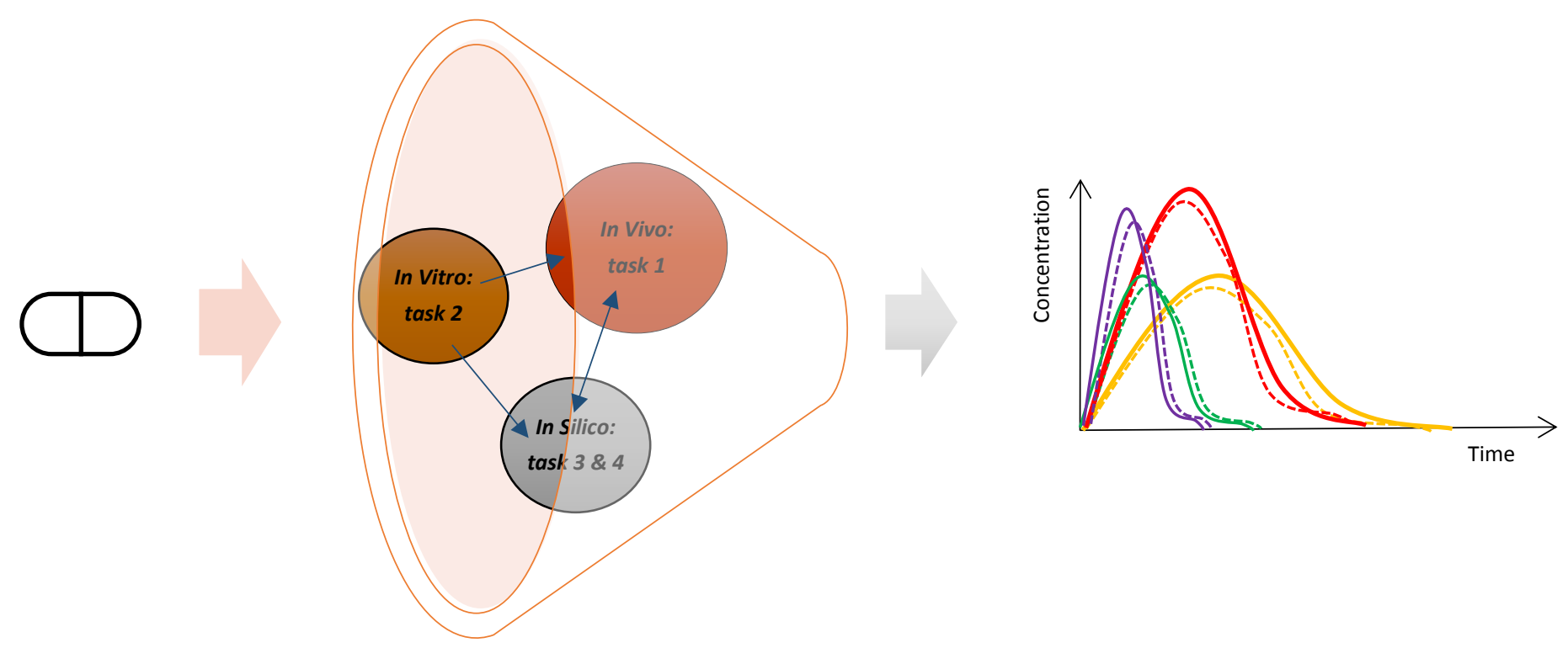

- Observed data

------ Predicted data

Volunteer 1

Volunteer 2

Volunteer 3 - visit 1

Volunteer $3-$ visit 2 


\section{Keywords}

in vivo dissolution; bioequivalence; bioavailability; oral absorption; MRI; manometry;

computational fluid dynamics

Abstract

Over the past decade, formulation predictive dissolution (fPD) testing has gained increasing attention. Another mindset is pushed forward where scientists in our field are more confident to explore the in vivo behavior of an oral drug product by performing predictive in vitro dissolution studies. Similarly, there is an increasing interest in the application of modern computational fluid dynamics (CFD) frameworks and high-performance computing platforms to study the local processes underlying absorption within the gastrointestinal (GI) tract. In that way, CFD and computing platforms both can inform future PBPK-based in silico frameworks and determine the GI-motility-driven hydrodynamic impacts that should be incorporated into in vitro dissolution methods for in vivo relevance. Current compendial dissolution methods are not always reliable to predict the in vivo behavior, especially not for biopharmaceutics classification system (BCS) class 2/4 compounds suffering from a low aqueous solubility. Developing a predictive dissolution test will be more reliable, cost-effective and less timeconsuming as long as the predictive power of the test is sufficiently strong. There is a need to develop a biorelevant, predictive dissolution method that can be applied by pharmaceutical drug companies to facilitate marketing access for generic and novel drug products. In 2014, Prof. Gordon L. Amidon and his team initiated a far-ranging research program designed to integrate (1) in vivo studies in humans in order to further improve the understanding of the intraluminal processing of oral dosage forms and dissolved drug along the gastrointestinal (GI) tract, (2) advancement of in vitro methodologies that incorporates higher levels of in vivo relevance and (3) computational experiments to study the local processes underlying dissolution, transport and absorption within the intestines performed with a new unique CFD based framework. Of particular importance is revealing the physiological variables determining the variability in in vivo dissolution and GI absorption from person to person in order to address (potential) in vivo $\mathrm{BE}$ failures. This paper provides an introduction to this multidisciplinary project, informs the reader about current achievements and outlines future directions. 


\section{Introduction}

Predicting the in vivo biopharmaceutical performance of oral drug products remains challenging, not only because of the properties of the drug but even more because of the drug product itself. In 2014, the University of Michigan was financially supported by the FDA (i) to explore the gastrointestinal (GI) processes that underlie the inter-/intra-subject systemic variability after administration of an oral drug product (task 1) (Hens et al., 2017a; Koenigsknecht et al., 2017; Yu et al., 2017, 2016) (ii) to advance the development of in vivo-relevant in vitro dissolution testing (task 2) (Kostewicz et al., 2014) and (iii) to develop and apply first-principles mathematical models and a new 'Computational Fluid Dynamics - Particle Model (CFD-PM)' framework to study local processes that underlie absorption within the intestines (tasks 3-4) (Brasseur et al., 2017; Mudie et al., 2015; Wang et al., 2015, 2012). Task 1 of the project initially focused on clinical aspiration/motility studies in healthy volunteers, the main population for in vivo bioequivalence (BE) studies. The GI and plasma concentrations of the weakly acidic drug ibuprofen (Biopharmaceutics Classification System (BCS) class IIa) (Amidon et al., 1995; Tsume et al., 2014) were measured after oral administration of an immediate-release (IR) tablet of the reference drug product (Shreveport, LA; Dr. Reddy's - IBU Ibuprofen Tablets, USP, 800 mg) (Hens et al., 2017a; Koenigsknecht et al., 2017). In parallel, motility pressure events were recorded with water-perfused manometry. The tablet was single-dose administered in fasted state conditions with a glass of water and in fed state conditions with a liquid meal (Pulmocare ${ }^{\circledR}$, Abbott Nutrition (Lake Forest, IL)) and a glass of water on separate study days. These data served as a reference to develop a formulation predictive (fPD) dissolution apparatus under design to (i) reflect in vivo performance of an oral drug product and (ii) explain potential failures in in vivo bioequivalence (BE) studies. More future aspiration/motility studies will be performed in order to extrapolate the predictive power of the GIS to different drug compounds (i.e., differences in physicochemical/biopharmaceutical properties). By replacing in vivo BE studies by fPD studies, the landscape of oral drug development will be drastically reshaped, both for the development of new as generic drug products. The costs for companies to authorize generic drug products is immense since more than $85 \%$ of all patients in the US are treated with generic drug products (Lennernäs et al., 2017a; Lionberger et al., 2013). Cook and Bockbrader calculated that 22-38 million dollars per year per generic 
drug product could be saved by making use of the BCS-based biowaivers for BCS class I drugs, assuming that $25 \%$ of BE studies are for class I drugs (Cook and Bockbrader, 2002). The same development cost savings can be applied to BCS class III drugs assuming $25 \%$ of BE studies are for class III drugs. Besides financial investment costs, in vitro studies assess drug product performance more directly compared to conventional pharmacokinetic (PK) studies in which drug absorption is measured indirectly for locally acting drugs (Polli, 2008). This is of particular significance, for example, in the case of oral drug products intended for local therapeutic action in the GI tract, where it is more difficult to connect the therapeutic effect to the plasma concentrations of the drug in the systemic circulation (e.g., sulfasalazine, 5-aminosalicylic acid). Drug product performance can be assessed and evaluated by in vitro dissolution and absorption experiments carried out both for the RLD and generic drug product. In vitro dissolution methods can also provide more rapid results, without the need of a clinician or willing subjects. This is another reason to replace in vivo BE studies by in vitro studies; as stated in the 21 CFR 320.25(a) in the USA (U.S. Government Printing Office, 2003), "no unnecessary human testing should be performed". This code states "the basic principle in an in vivo bioavailability study is that no unnecessary human research should be done."

To summarize, there is an immense need to develop biorelevant, predictive dissolution methods for application by generic and novel drug companies to facilitate marketing access for a wide-spread variety of oral drug products in terms of formulation type and active pharmaceutical ingredients (APIs) from various BCS classes (Polli et al., 2008, 2004). In 2014, Prof. Gordon Amidon and his team initiated in vivo studies in humans to analyze the complexities in the intraluminal behavior of oral dosage forms in the GI tract. Prof. Gregory Amidon lead an effort to develop in vivo relevant in vitro testing, and Prof. Brasseur lead the development and application of advanced, new mathematical and computer models. Of particular importance is the determination of the physiological variables that underlie intra-/intersubject variabilities in in vivo dissolution and absorption to address (potential) in vivo BE failures. This manuscript summarizes the current state of this multidisciplinary project.

\section{General outline of the $21^{\text {st }}$ Century BE project and selected tasks}

The strategy of this FDA-funded project is to create an fPD device that can predict the in vivo performance of an oral drug product (Task 2). In order to build this robust set of model, in vivo data are 
required (Task 1). In addition, a new computational fluid dynamics (CFD) high-performance computing framework was developed and applied in two series of computational experiments (Tasks 3-4). To schedule all in vivo experiments for this ongoing project, the different objectives are categorized within different tasks:

- Task 1: In vivo studies: Intraluminal profiling, manometry, magnetic resonance imaging (MRI)

- Task 2: In vitro studies: Gastrointestinal Simulator (GIS)

- Task 3 \& 4: In silico studies: mass transport modeling (MTM) \& computational fluid dynamics - particle model (CFD-PM) 
Task 1 is an in vivo work package focused on mechanistic in vivo biopharmaceutical studies with the focus on intraluminal profiling of drug concentrations in parallel with simultaneously analyzing motility events along the human GI tract. The GI tract is a complex and dynamic organ with large regions, making it a challenge to assess in detail (Grønlund et al., 2017). The first study was an in vivo aspiration study that was successfully completed in 37 healthy volunteers (Hens et al., 2017a; Koenigsknecht et al., 2017). A customized multi-channel manometric catheter was intubated by mouth and positioned in the human GI tract of all participating subjects. After oral administration of an 800 mg ibuprofen tablet, GI fluids were aspirated from the stomach, duodenum and jejunum. In parallel, blood samples were taken to measure plasma exposure over time of the study drug. These studies were conducted in fasted $(\mathrm{n}=20)$ and fed states $(\mathrm{n}=17)$. The results of this study indicated that the in vivo drug dissolution is much slower than what is observed using traditional in vitro methods, which appears to be primarily due to the low buffer capacity of the intestinal fluids. Moreover, a clear linear correlation was observed between the time when a high burst of phase III contractile waves was present and the maximal concentration of ibuprofen in plasma (plasma $C_{\max }$ ) (Hens et al., 2017a; Talattof et al., 2016). This study demonstrated the importance of motility and gastric emptying versus $\mathrm{pH}$ and buffer capacity as major determinants responsible for the systemic exposure of an orally-administered drug product.

To have an adequate reflection of fluid distribution along the GI tract, a phenol red solution $(0.1$ $\mathrm{mg} / \mathrm{mL}$ ) was poured into the glass of water that was administered to the subjects in the same study. In this case, we were able to monitor GI fluid changes related to dilution, secretion, and absorption in fasted and fed state conditions. Phenol red is a BCS class 3 compound with negligible absorption characteristics (Dahlgren et al., 2017). Phenol red concentrations were analyzed in the aspirated fluids and will serve as reference data in order to develop an MTM that can reflect the real-life situation of GI fluid changes throughout the entire GI tract (stomach, duodenum and jejunum), respecting the changes in GI physiology in fasted and fed state conditions.

Additional insights into contractile events along the GI tract will be explored by MRI studies in the coming year at the University of Nottingham (Figure 1). MRI is being used to quantify the time courses of the volumes of freely mobile fluid in the stomach and bowel together with GI motility, within the same study session, in healthy adult participants (Khalaf et al., 2015; Mudie et al., 2014; Murray et 
al., 2017). In addition to volumes, several MRI studies have already quantified motility by (i) using a registration algorithm that provided a global motility metric in arbitrary units (Menys et al., 2013) or by (ii) administering nonabsorbable fluid to monitor small bowel contractions (Wakamiya et al., 2011). Current work is cross-validating the MRI small bowel motility protocol with the one used previously at the University of Michigan (Hens et al., 2017a). The ultimate aim is to show that invasive manometric intubation methods can be replaced by noninvasive MRI methods. Future plans will include correlating plasma drug time courses with GI fluid volumes and motility in the unprepared and undisturbed (i.e., not intubated) bowel. Although not a main outcome of the primary validation study, the MRI data are also expected to enable identification contractile phases of the migrating motor complex (MMC). The ability of MRI to monitor the entirety of the GI tract and to detect non-occlusive contractions of the lumen should also prove that the MRI methods could actually provide superior information to that obtained under conventional perfused manometry (Wright et al., 1999). 


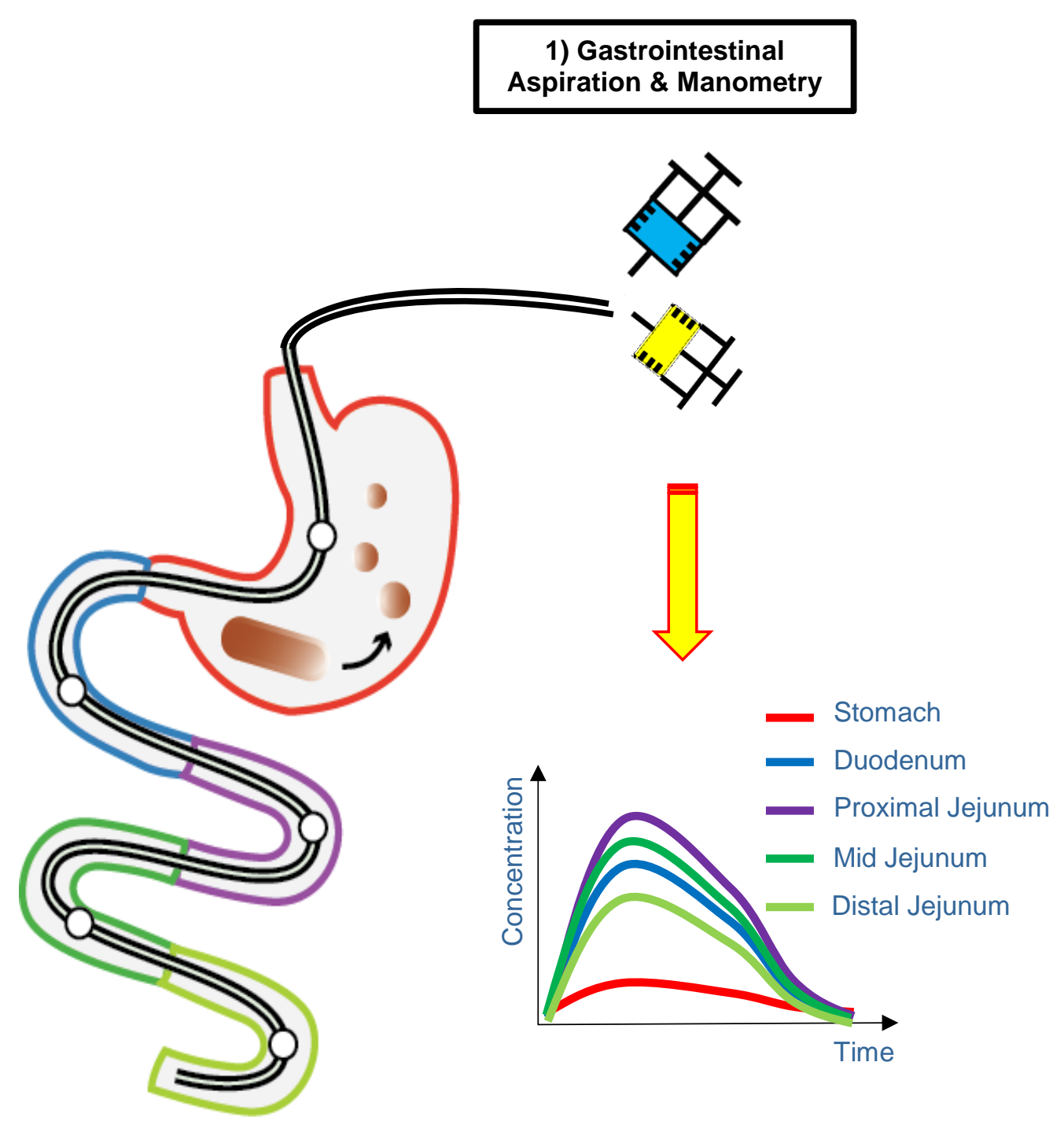

2) MRI Scanning

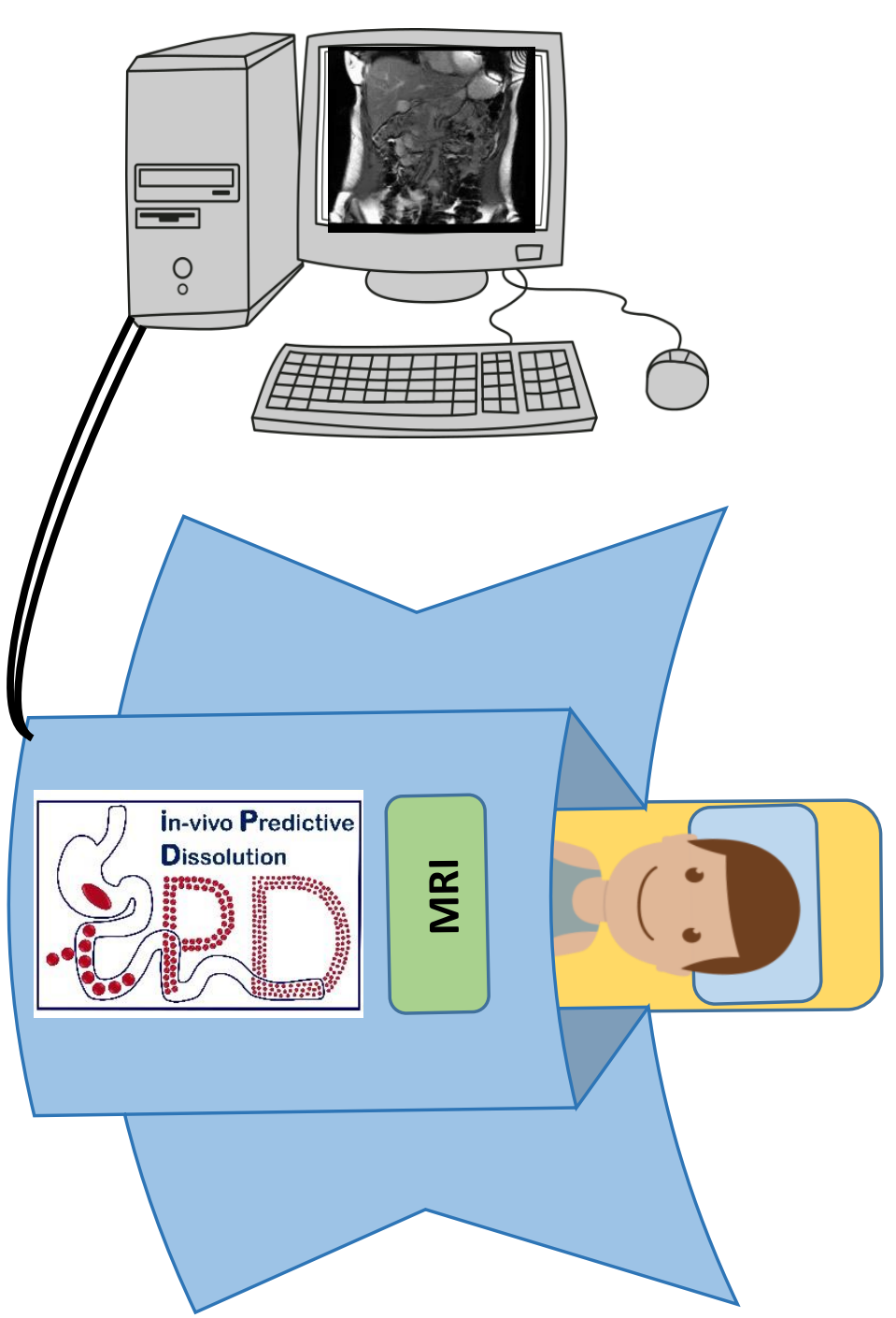

Figure 1: Representative illustration of the 1) aspiration-motility study and 2) MRI scanning study that are performed in task 1 of this BE project. 
Task 2 focuses on biorelevant in vitro studies that reflect in vivo behavior of the oral drug product, as quantified in task 1 . There are many in vitro models described in the literature and these tools are subject to optimization/ validation throughout different biopharmaceutical projects at the moment. These models can be classified as rather simple, static models (e.g., USP 2/4 models) towards more complex and dynamic models (e.g., ASD model, BioGIT, Transfer Model and TIMagc) (Carino et al., 2006; Kostewicz et al., 2014; Kourentas et al., 2016; Matsui et al., 2016; Verwei et al., 2016). Under development is the 'Gastrointestinal Simulator' (GIS), consisting of gastric, duodenal and jejunal chambers (Figure 2).

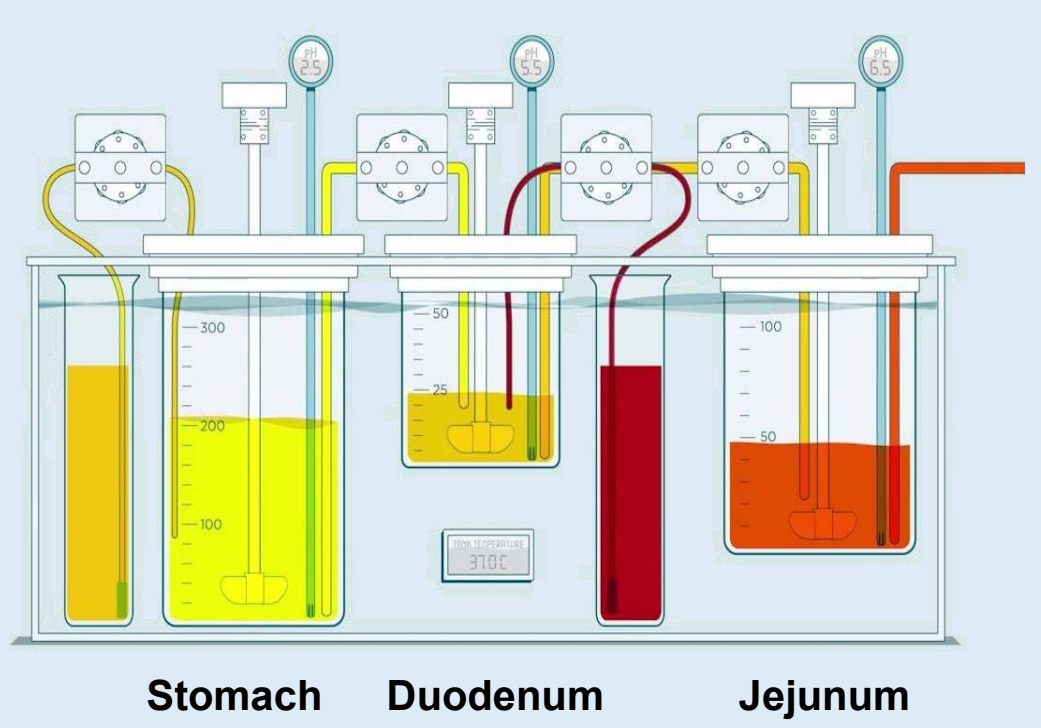

Figure 2: Setup of the GIS consisting of a gastric, duodenal and jejunal chamber. Gastric and duodenal secretions are simulated as well. The $\mathrm{pH}$ can be measured during the entire experiment in all compartments. Figure adapted from Hens et al., (2018) - Copyright Elsevier 2018.

The major advantage of this user-friendly model is a directly controlled adjustment of parameters relevant to in vivo dissolution and absorption. For example, rapid transfer of gastric emptying has been observed in humans: gastric half-life values from 4 - 13 min in fasted state conditions following a first-order kinetic process (Hens et al., 2014; Tsume et al., 2017). To confirm, Mudie and colleagues demonstrated the same fast, first-order gastric emptying of $240 \mathrm{~mL}$ of water in 12 healthy subjects by performing MRI scanning (Mudie et al., 2014). These scenarios can be implemented in the GIS by controlling the rates of the individual peristaltic pumps and the transfer between the gastric and duodenal chambers, duodenal to jejunal chambers, as well as the separate gastric and duodenal secretions. To 
simulate absorption, an artificial membrane was recently developed. The creation of an absorptive sink by working with a polydimethylsiloxane (PDMS) membrane to simulate oral drug absorption has recently been investigated and approved (Sinko et al., 2017). The customized PDMS membrane showed biomimetic properties related to the intestinal membrane because of similarities in small molecule transport, such as mechanism, ionization selectivity and lipophilicity. The impact of sink conditions on intraluminal concentrations can be significant as demonstrated by Bevernage and colleagues (Bevernage et al., 2012). In this work, the precipitation kinetics of loviride were evaluated after induction of different supersaturated solutions at the apical side of the Caco- 2 setup. The impact of an absorptive environment was evaluated in presence and absence of sink conditions (i.e., by parafilm-sealing the insert and in absence of a cell monolayer). Also, the micellar encapsulation of a lipophilic drug can hamper the intestinal absorption as shown for fenofibrate in man and in vitro (Buch et al., 2009; Hens et al., 2015; Sironi et al., 2017). Solubilization of the drug will be enhanced in the presence of colloidal structures such as (mixed) micelles and vesicles, but permeation can be hampered. Only the free fraction (i.e., not encapsulated) will be available for intestinal absorption (Dahan et al., 2016). In that case, respecting the dissolution/permeation interplay is indispensable to make the right predictions towards the in vivo performance of the drug. Implementation of the PDMS membrane in the GIS device is currently ongoing and will help us to directly look at formulation performance considering the dissolution/permeation interplay.

The volumes and dissolution media can be adjusted in order to resemble different scenarios consistent with the protocol of the clinical studies. Various studies have been successfully conducted to predict the in vivo performance of orally administered BCS class IIb compounds (Matsui et al., 2017, 2016, 2015; Tsume et al., 2015). The obtained in vitro dissolution profiles can be applied as an input for a physiologically-based pharmacokinetic (PBPK) model describing absorption, distribution and clearance of the drug of interest. The predicted plasma profiles can be directly compared with observed in vivo plasma data. In this way, the GIS device can be evaluated and validated. Moreover, if intraluminal concentration-time profiles of the drug of interest are quantified (e.g., gastric and intestinal determined concentrations), a direct validation of the GIS can be established by directly comparing the in vitro 
dissolution profiles with the measured intraluminal concentrations. Optimization and validation of the current GIS model will lead, in the end, to a newer version. Currently, attention has been directed at vessel shape and stirrer design to reproduce the mixing conditions as observed in vivo. Average shear rates are simulated in different vessel designs to provide an optimal design using different kinds of stirrers in order to select the right vessel and stirrer that closely mimics the in vivo shear rates (Baxter et al., 2006, 2005; Kukura et al., 2004). For instance, Figure 3 depicts the CFD in the gastric (A) and duodenal (B) chamber of the GIS device as well as in the (C) USP II dissolution apparatus with a stirring paddle (100 rotations per minute). 


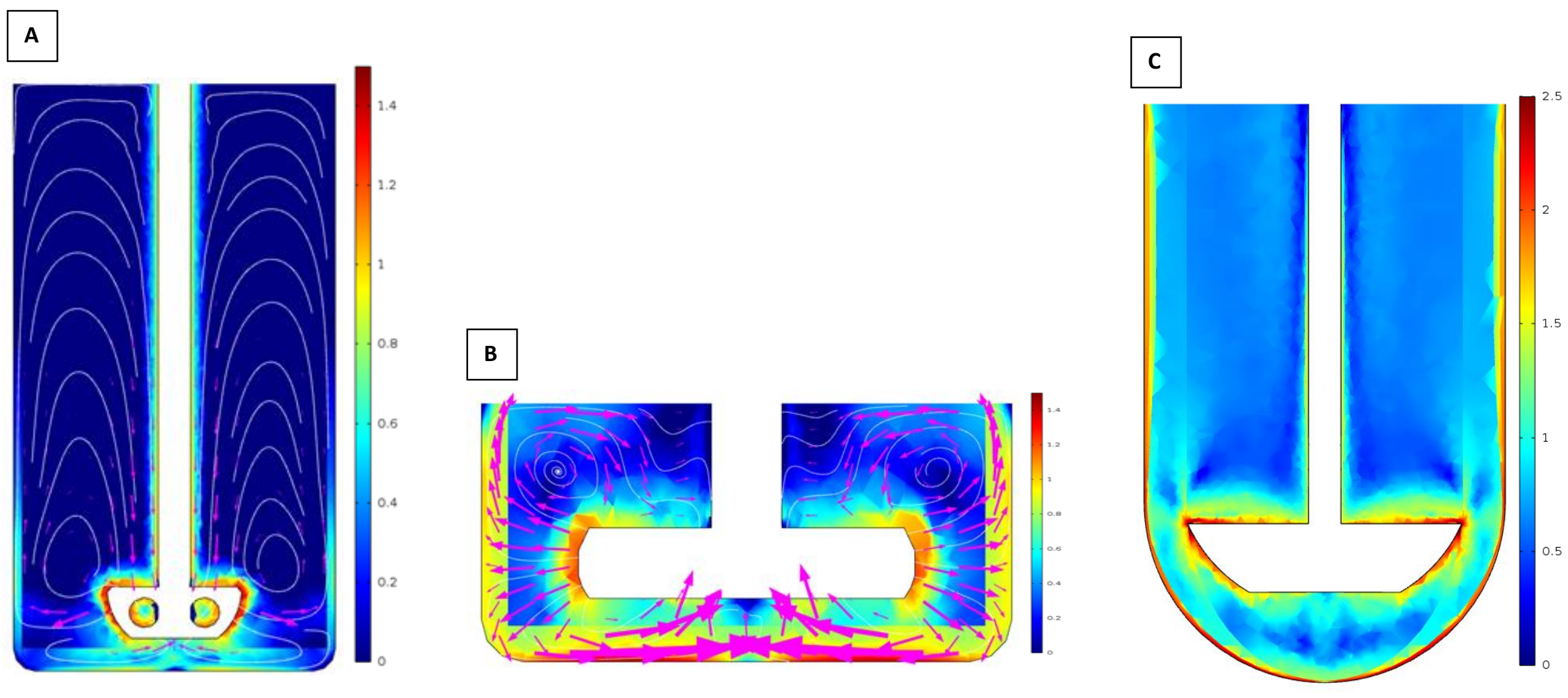

Figure 3: Logarithmic-transformed shear rate values (A) in the stomach compartment of the GIS (25 rotations per minute), (B) in the duodenal compartment of the GIS (25 rotations per minute) and (C) in the USP II dissolution device (100 rotations per minute).USP II dissolution apparatus with stirring paddle (100 rotations per minute). Simulations were performed by applying COMSOL Multiphysics ${ }^{\circledR}$ (Burlington, MA). Partially adopted from Salehi et al., (2017). 
Besides hydrodynamics, selection of buffer species (bicarbonate versus phosphate) and buffer concentrations are subject of interest as the low buffer capacity of the aspirated fluids was responsible for the dynamic pH range along the GI tract (Krieg et al., 2015, 2014). Especially for enteric-coated dosage forms, physiological bicarbonate buffers proved to be more discriminative with respect to the drug release behavior. Moreover, the adoption of inappropriate buffer media for in vitro BE studies would likely cause a failure in achieving an in vitro-in vivo correlation (IVIVC) for oral drug products. Therefore, we will investigate the impact of the buffer species on the drug release for different dosage forms during this project.

Task 3 centered on the development and application of mathematical models of the rate of release of drug molecules from individual small spherical drug particles in a 'hierarchical' framework that allows for extensions that can include adjustments to dissolution rate from, among other effects, hydrodynamic (i.e., flow) influences on single-particle dissolution rate. In task 4 we developed a computational fluid dynamics (CFD) framework with an embedded mathematical 'particle model' (PM) that has the 'hierarchical mathematical model' of task 3 at its core (Wang et al., 2015, 2012). The development of the hierarchical mathematical model framework included a major advance in modeling and analysis of hydrodynamic influences on dissolution rate as well detailed analysis of the consequences of variations in solid drug concentration and solubility, particle size distribution and the confinement of drug molecules by vessel boundaries in in vitro devices with impermeable containers. This new understanding was embedded within a CFD-PM framework based on the lattice-Boltzmann numerical method (Wang et al., 2010). Using the CFD-PM we have designed and carried two classes of computational experiments on 'high-performance computing' (HPC) platforms, as briefly described below ('HPC' currently implies the application of multi-processor computers requiting parallel processing methods.).

Figure 4 illustrates key issues addressed using the CFD-PM, specifically drug release, transport and absorption within localized fluid-filled 'pockets' of the GI tract. The central point illustrated in this figure is that absorption of drug molecules can take place only if they are presented to the mucosal epithelium, so that absorption depends on the mechanisms underlying the rate at which drug molecules 
are transported to the intestinal wall from the bulk fluid in relation to the rate at which drug molecules are released into the bulk. This relationship is driven by the complex array of motility-driven hydrodynamic motions within the stomach and intestines that varies with the physiological and function state of the GI tract. In the fed state, when GI function is directed at nutrient absorption, regular 3/min peristaltic contractions in the gastric antrum coordinate with pyloric opening to allow transport of gastric content into the duodenum at a controlled rate. The dominant motility of the duodenum and jejunum in the fed state is peristalsis, both forward and reversed for transport and mixing, and segmental contractions that enhance mixing locally (Ailiani et al., 2009; Banco, 2010). The fasting state is characterized by lack of nutrients in the GI tract, and therefore lack of function associated with nutrient absorption. Motility in the fasting state is characterized by the MMC which occurs in roughly three phases that repeat with a period typically in the range of 60-90 minutes. Whereas phase I is characterized by overall quiescence, phases II and III involve motility patterns that can be disorganized or organized and which involve peristalsis locally at very different spatial and temporal scales. We have begun to study these relationships in the jejunum using the new CFD-PM developed under the current FDA program.

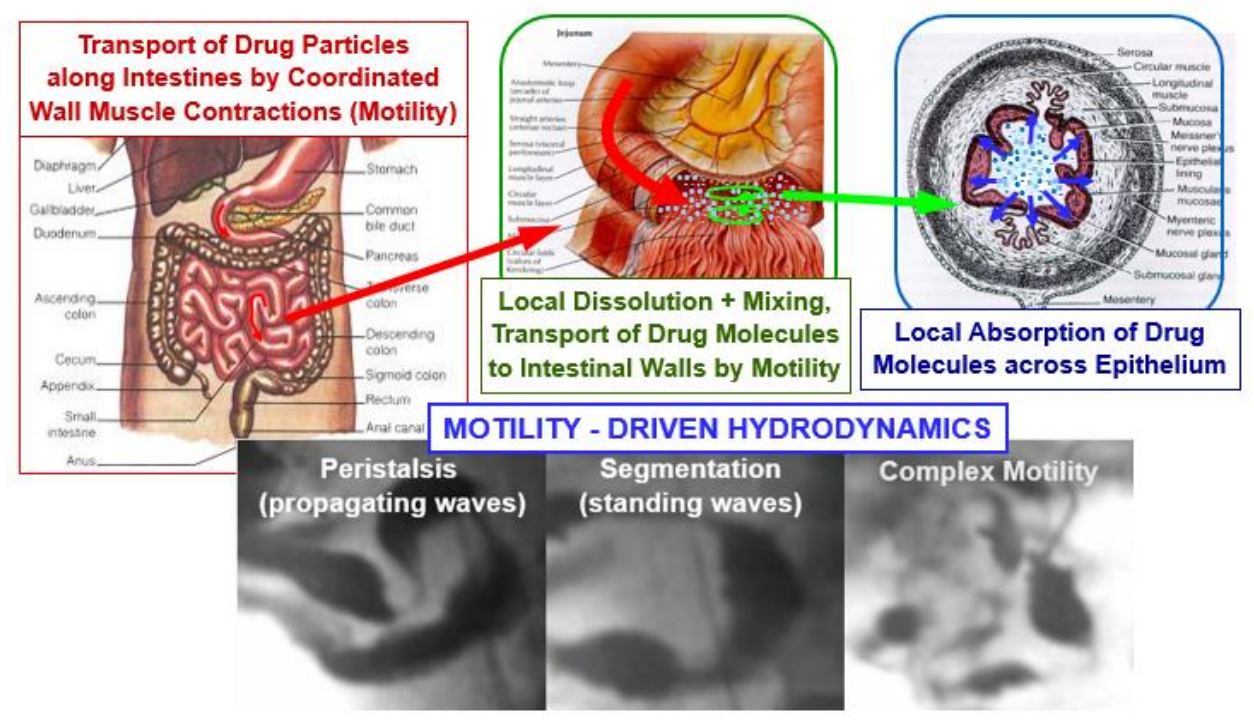

Figure 4: The role of intestinal transport and contractile patterns (motility) on motility in drug release (dissolution), drug particle and molecular transport, and drug absorption. The upper images illustrate global transport of intestinal content (upper left image) by peristaltic contractile patterns (left lower image). Peristalsis both transports and mixes drug particles (middle upper image) as drug molecules are released and mixed and absorbed (upper right image) within localized "pockets" of fluid that change in time by the axially moving local contractile patterns (lower left image). In addition, standing wave "segmentation" motility patterning (lower middle image) predominantly mix drug particles and molecule locally within pockets, and increases lateral transport of drug molecules to the mucosal surface (upper right image), where they can be absorbed. Whereas peristalsis and segmentation are the more common canonical motility patterns, more complex patterns, with mixes of peristalsis and segmentation, can occur (bottom right image), as are periods with little or no motility. Each different motility pattern impacts hydrodynamic contributions to dissolution and transport differently. 
The CFD-PM framework is designed to predict the development and spatio-temporal evolution of the local concentration field in response to the release of drug molecules from the surfaces of thousands of drug particles of varying diameter. Both the particles and the drug molecules are carried by the local velocity field resulting from spatio-temporal contractions of the intestinal (or gastric) lumen. To date, two sets of computational experiments for release, transport and absorption of ibuprofen (a class II weak acid with $\mathrm{pKa} \sim 4.4$ ) have been performed. All experiments carried out to date have applied a geometry model for peristaltic motility using data from Mudie et al., (2014) to estimate 'small' and 'large' intestinal pocket volumes within the true fasting state in vivo range of 2.0 and $12.1 \mathrm{~mL}$, respectively (Figure 5).

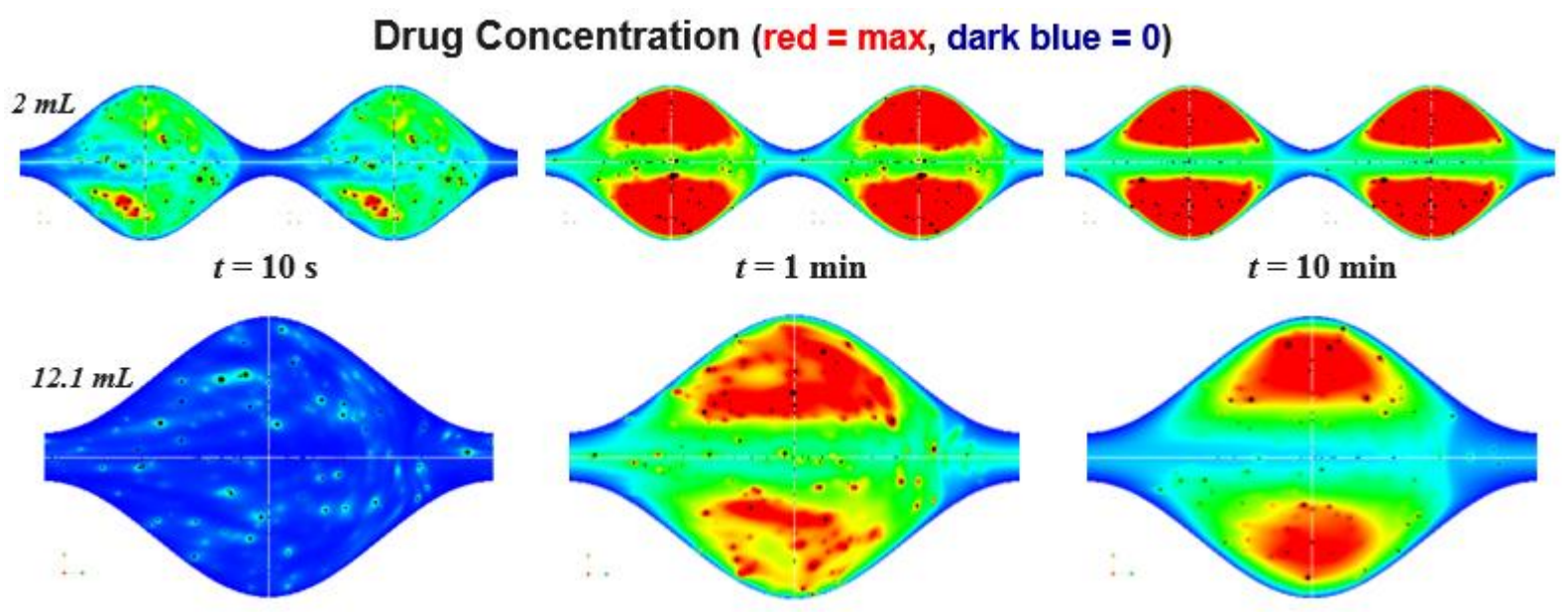

Figure 5: Drug concentration at cross section of the intestinal pockets for large and small volumes and with the maximum permeability boundary condition at different times during the transition from an initial state when no drug has been released into the bulk fluid to a quasisteady state period when the structure of the concentration field remains roughly constant in time. Maximum concentration is shown in dark red and zero concentration in dark blue in a frame-of-reference moving with the peristaltic waves. The quasi-steady period starts earlier with smaller pocket volumes and lasts for 50-60 minutes before a long-lasting final period over which all particles have dissolved and all released drug molecules have been absorbed (end simulation run: $180 \mathrm{~min}$ ). Both drug concentration and particle concentration (not shown) develop a highly heterogeneous distribution as a result of the motility-forced velocity field. In this "max permeability" scenario, absorption results from a balance between transport (advection) into a central "bubble" of recirculating flow and diffusion of drug molecules from the recirculating bubble across a narrow fluid layer to the mucosal surface.

In Figure 5 we show the center plane of trains of peristaltic waves at different times during the initial period in drug release from 4216 particles over a range of particle sizes measured from the IR ibuprofen tablet (Shreveport, LA; Dr. Reddy's - IBU - Ibuprofen Tablets, USP, 800 mg). From one of the very few studies with in vivo data from which peristaltic wave speeds in the fasting state can be 
estimated (Castedal and Abrahamsson, 2001), we estimated peristaltic wave speed for peristaltic events in the phase III MMC period to be of order $1 \mathrm{~cm} / \mathrm{s}$. During this period a highly heterogeneous distribution of drug concentration develops, largely driven by the details of the pocket velocity field driven by peristalsis. Drug absorption, which takes place at the surface, results from an interaction between bulk transport of particles and concentration by the velocity within the 'trapped' fluid regions, shown in Figure 5 by the region with the highest drug concentration (red area), and diffusion of drug molecules from this trapped region to the mucosal surface. We find that a quasi-steady state period is established within roughly $1 \mathrm{~min}$ for the $2 \mathrm{~mL}$ pocket and within roughly $10 \mathrm{~min}$ for the $12.1 \mathrm{~mL}$ peristaltic pockets. We also find that hydrodynamic enhancement of dissolution and absorption rate from slip velocity (convection) is only a $1-2 \%$ effect while the shear-rate effect is very strong, causing a $40-50 \%$ increase in dissolution and absorption rate compared to pure diffusion. A number of important mechanistic processes and their interactions uncovered and analyzed will be discussed in forthcoming scientific papers. 


\section{Concluding remarks \& Future Perspectives}

The intensive collaborations among gastroenterologists, statisticians, chemical engineers, pharmaceutical scientists and regulatory authorities are indispensable in this project in order to achieve our goals (Table 1). 


\section{UNIVERSITY OF MICHIGAN}

\section{Pharmaceutical Sciences}

Gordon L. Amidon, Ph.D., P.I.

Gregory E. Amidon, Ph.D., Co-I

\section{Duxin Sun, Ph.D.}

Yasuhiro Tsume, Associate Research Scientist

Paulo Paixão, Visiting Scientist

Marival Bermejo, Visiting Scientist

Naír Rodríguez-Hornedo, Ph.D.

Bart Hens, PostDoc, Visiting Scientist

Raimar Löbenberg, Visiting Scientist

Jozef Al-Gousous, PostDoc

Arjang Talattof, Consultant

Nicholas Job, Grad Student

Patrick Sinko, Grad Student

Kai Wang, Grad Student

Alex Yu, Grad Student

Meagan Dean, Undergrad Student

Mark Koenigsknecht, PostDoc

Gail Benninghoff, Program Manager

\section{University Hospitals Michigan}

William L. Hasler, M.D., Ph.D

Jason R. Baker, Ph.D.

Ann Frances, Clinical Research Coordinator

Jeffrey Wysocki, RN

Allen Lee, M.D.

\section{Statistics}

Kerby Shedden, Ph.D., Co-I

Joseph Dickens, Grad Student

\section{Chemical Engineering}

Robert Ziff, Ph.D., Co-I

Niloufar Salehi, Grad Student

\section{Grants Management}

Jan Mitchell, Grants Management

\section{UNIVERSITY OF COLORADO}

James Brasseur Ph.D., Co-I

Farhad Behafarid, Ph.D.

\section{UNIVERSITY OF NOTTINGHAM (MRI)}

\section{Luca Marciani, Ph.D. Co-I}

Nichola Abrehart, Research Fellow

Khaled Heissam, Senior Research Fellow

Jeff Wright, GI Physiologist

Maura Corsetti, gastroenterologist

Caroline Hoad, MRI Physicist

Penny Gowland, MRI Physicist

\section{U.S. FDA}

Jianghong Fan, DQMM/ORS/OGD/CDER

Liang Zhao, DQMM Division Director

Robert Lionberger, ORS Office Director

Minori Kinjo, ORS RIHSC PM

Zongming Gao, Chemist

John Duan - Pharmacologist

Hong Wen - Chemist

Dajun Sun - DQMM/ORS/OGD/CDER

Stephanie Choi - Acting Associate Director for Science

\section{COLLEAGUES AND ASSOCIATES}

David Fleisher (UM, US)

Shinji Yamashita (Setsunan, JP)

Peter Langguth ( JGU Mainz, DE)

Hans Lennernäs (Uppsala, SE) 
Similar efforts are ongoing in European projects, such as OrBiTo (http://www.orbitoproject.eu) (Lennernäs et al., 2014). This project is funded by the Innovative Medicines Initiative (IMI) and the European Federation of Pharmaceutical Industries and Associations (EFPIA). The same philosophy/vision is centralized in this project: "Transform our ability to predict the in vivo performance of oral drug products across all stages of drug development". This six-year ongoing project (2012-2018) demonstrated the closed interaction and collaboration between academic institutions and pharmaceutical companies in order to increase the predictive power of biorelevant in vitro/in silico models that are frequently used in preclinical formulation development (industry-driven project).

In 2016, The PEARRL project kicked off and received funding under the Marie SkłodowskaCurie Innovative Training Networks of the Horizon 2020 program of the European Union. The members of this project consist of 9 beneficiaries and 10 partner organizations from 8 different countries, closely collaborating on biopharmaceutical themes with a regulatory link. All partners employ $15 \mathrm{PhD}$ researchers that will be trained/prepared to mature scientists after an intensive training of three years in an academic, industrial and regulatory setting (http://www.pearrl.eu).

More recently, a new action (The European Network on Understanding Gastrointestinal Absorption-related Processes (UNGAP)) got set up to manage a multidisciplinary network of scientists aiming to advance the field of intestinal drug absorption by concentrating on four major challenges: (i) differences between specific patient populations, (ii) regional differences along the GI tract, (iii) the intraluminal behavior of advanced formulations, and (iv) food-drug interactions. This European cooperation in science and technology (COST) action seeks to connect specialists in different pharmaceutical/medical fields in order to unravel the black boxes of the GI tract. This action is financially supported by the Horizon 2020 Framework program of the European Union (http://www.ungap.eu). 
Regarding all these ongoing projects worldwide, a broad discussion is recently started for an International Conference on Harmonization (ICH) initiative on BE. It is an ongoing process to define regional global differences on IVIVC and BE (Lennernäs et al., 2017b). For instance, the ingested volume of water co-administered in clinical studies with the oral drug product differ between continents (240 mL for FDA and EMA versus $150 \mathrm{~mL}$ for PDMA) (European Medicines Agency, 2010; Food \& Drug Administration, 2015; Pharmaceuticals and Medical Devices Agency, 2012). Recent studies in Japan and Belgium showed that barely anyone takes their oral medicines with a full glass of water, but rather with $88 \mathrm{~mL}$ (Japan; n=512) or $125 \mathrm{~mL}$ (Belgium; n=1977) ( Hens et al., 2017b; Sugihara et al., 2015).

The human studies as described in task 1 will result in a comprehensive human database in terms of measured drug concentrations along the GI tract and measured impact of the dynamic physiology on these concentrations. For the next years, the focus will encompass MRI studies that will investigate and quantify dynamic and physiological aspects of the GI tract: fluid volumes, motility and transit. In a second step, the MRI study will be extended to oral administration of a drug product and blood sampling under MRI monitoring of the bowel. This approach opens horizons to look at oral drug product processing in any kind of population: pregnant/childbearing/lactating women, specific patient populations, children and elderly. To conclude, because of this multidisciplinary approach, we will extend our fundamental knowledge of the GI tract to deliver a formulation predictive dissolution apparatus that will support generic and new drug product development.

\section{Acknowledgments}

This work is supported by grant \# HHSF223201510157C and grant \# HHSF223201310144C by the U.S. Food and Drug Administration (FDA). This report represents the scientific views of the authors and not necessarily that of the FDA. Authors have no conflicts of interest to declare. Bart Hens wants to acknowledge the Internal Funds of KU Leuven (KU Leuven - Internal Funds PDM/17/164). More information about the project can be found on: http://www.ddfint.org/odd-2018/

\section{References}

Ailiani, A.C., Neuberger, T., Brasseur, J.G., Banco, G., Wang, Y., Smith, N.B., Webb, A.G., 2009. Quantitative analysis of peristaltic and segmental motion in vivo in the rat small intestine using dynamic MRI. Magn Reson Med 62, 116-126. https://doi.org/10.1002/mrm.21982 
Amidon, G.L., Lennernäs, H., Shah, V.P., Crison, J.R., 1995. A theoretical basis for a biopharmaceutic drug classification: the correlation of in vitro drug product dissolution and in vivo bioavailability. Pharm. Res. 12, 413-420.

Banco, G.G., 2010. Multi-scale Fluid Mechanics of Nutrient Absorption in the Small Intestine Analyzed with $2 \mathrm{~d}$ and $3 \mathrm{~d}$ Lattice Boltzmann Models. Pennsylvania State University.

Baxter, J.L., Kukura, J., Muzzio, F.J., 2006. Shear-induced variability in the United States Pharmacopeia Apparatus 2: modifications to the existing system. AAPS J 7, E857-864. https://doi.org/10.1208/aapsj070483

Baxter, J.L., Kukura, J., Muzzio, F.J., 2005. Hydrodynamics-induced variability in the USP apparatus II dissolution test. Int J Pharm 292, 17-28. https://doi.org/10.1016/j.ijpharm.2004.08.003

Bevernage, J., Brouwers, J., Annaert, P., Augustijns, P., 2012. Drug precipitation-permeation interplay: supersaturation in an absorptive environment. Eur J Pharm Biopharm 82, 424-428. https://doi.org/10.1016/j.ejpb.2012.07.009

Brasseur, J.G., Behafarid, F., Wang, Y., Mudie, D., Amidon, G.L., 2017. Hydrodynamic Influences on Drug Dissolution and Absorption In Vitro and In Vivo, quantified with Mathematical Models and Computer Simulation. Presented at the 6th Pharmaceutical Sciences World Congress (FIP PSWC 2017), Stockholm, Sweden.

Buch, P., Langguth, P., Kataoka, M., Yamashita, S., 2009. IVIVC in oral absorption for fenofibrate immediate release tablets using a dissolution/permeation system. J Pharm Sci 98, 20012009. https://doi.org/10.1002/jps.21576

Carino, S.R., Sperry, D.C., Hawley, M., 2006. Relative bioavailability estimation of carbamazepine crystal forms using an artificial stomach-duodenum model. J Pharm Sci 95, 116-125. https://doi.org/10.1002/jps.20495

Castedal, M., Abrahamsson, H., 2001. High-resolution analysis of the duodenal interdigestive phase III in humans. Neurogastroenterol. Motil. 13, 473-481.

Cook, J.A., Bockbrader, H.N., 2002. An Industrial Implementation of the Biopharmaceutics Classification System. Dissolution Technologies.

Dahan, A., Beig, A., Lindley, D., Miller, J.M., 2016. The solubility-permeability interplay and oral drug formulation design: Two heads are better than one. Adv. Drug Deliv. Rev. 101, 99-107. https://doi.org/10.1016/j.addr.2016.04.018

Dahlgren, D., Roos, C., Lundqvist, A., Tannergren, C., Langguth, P., Sjöblom, M., Sjögren, E., Lennernäs, H., 2017. Preclinical Effect of Absorption Modifying Excipients on Rat Intestinal Transport of Model Compounds and the Mucosal Barrier Marker 51Cr-EDTA. Mol. Pharm. 14, 4243-4251. https://doi.org/10.1021/acs.molpharmaceut.7b00353

European Medicines Agency, 2010. Guideline on the investigation of bioequivalence.

Food \& Drug Administration, 2015. Waiver of In Vivo Bioavailability and Bioequivalence Studies for Immediate-Release Solid Oral Dosage Forms Based on a Biopharmaceutics Classification System Guidance for Industry.

Grønlund, D., Poulsen, J.L., Sandberg, T.H., Olesen, A.E., Madzak, A., Krogh, K., Frøkjaer, J.B., Drewes, A.M., 2017. Established and emerging methods for assessment of small and large intestinal motility. Neurogastroenterol. Motil. 29. https://doi.org/10.1111/nmo.13008

Hens, B., Bermejo, M., Tsume, Y., Gonzalez-Alvarez, I., Ruan, H., Matsui, K., Amidon, G.E., Cavanagh, K., Kuminek, G., Benninghoff, G., Fan, J., Rodríguez-Hornedo, N., Amidon, G.L., 2018. Evaluation and optimized selection of supersaturating drug delivery systems of posaconazole (BCS class $2 \mathrm{~b}$ ) in the gastrointestinal simulator (GIS): An in vitro-in silico-in vivo approach. Eur J Pharm Sci. https://doi.org/10.1016/j.ejps.2018.01.039

Hens, B., Brouwers, J., Anneveld, B., Corsetti, M., Symillides, M., Vertzoni, M., Reppas, C., Turner, D.B., Augustijns, P., 2014. Gastrointestinal transfer: in vivo evaluation and implementation in in vitro and in silico predictive tools. Eur J Pharm Sci 63, 233-242.

https://doi.org/10.1016/j.ejps.2014.07.008 
Hens, B., Brouwers, J., Corsetti, M., Augustijns, P., 2015. Gastrointestinal behavior of nano- and microsized fenofibrate: In vivo evaluation in man and in vitro simulation by assessment of the permeation potential. Eur J Pharm Sci 77, 40-47. https://doi.org/10.1016/j.ejps.2015.05.023

Hens, B., Tsume, Y., Bermejo, M., Paixao, P., Koenigsknecht, M.J., Baker, J.R., Hasler, W.L., Lionberger, R., Fan, J., Dickens, J., Shedden, K., Wen, B., Wysocki, J., Loebenberg, R., Lee, A., Frances, A., Amidon, G., Yu, A., Benninghoff, G., Salehi, N., Talattof, A., Sun, D., Amidon, G.L., 2017a. Low Buffer Capacity and Alternating Motility along the Human Gastrointestinal Tract: Implications for in Vivo Dissolution and Absorption of lonizable Drugs. Mol. Pharm. 14, 42814294. https://doi.org/10.1021/acs.molpharmaceut.7b00426

Hens, B., Van Den Abeele, J., Rubbens, J., Keirsebilck, M., Roelens, J., Schreurs, C., Verheyen, K., Casteels, M., Laekeman, G., Augustijns, P., 2017b. Evaluation of real-life dosing of oral medicines with respect to fluid and food intake in a Dutch-speaking population. J Clin Pharm Ther 42, 467-474. https://doi.org/10.1111/jcpt.12535

Khalaf, A., Hoad, C.L., Spiller, R.C., Gowland, P.A., Moran, G.W., Marciani, L., 2015. Magnetic resonance imaging biomarkers of gastrointestinal motor function and fluid distribution. World J Gastrointest Pathophysiol 6, 140-149. https://doi.org/10.4291/wjgp.v6.i4.140

Koenigsknecht, M.J., Baker, J.R., Wen, B., Frances, A., Zhang, H., Yu, A., Zhao, T., Tsume, Y., Pai, M.P., Bleske, B.E., Zhang, X., Lionberger, R., Lee, A., Amidon, G.L., Hasler, W.L., Sun, D., 2017. In Vivo Dissolution and Systemic Absorption of Immediate Release Ibuprofen in Human Gastrointestinal Tract under Fed and Fasted Conditions. Mol. Pharm. 14, 4295-4304. https://doi.org/10.1021/acs.molpharmaceut.7b00425

Kostewicz, E.S., Abrahamsson, B., Brewster, M., Brouwers, J., Butler, J., Carlert, S., Dickinson, P.A., Dressman, J., Holm, R., Klein, S., Mann, J., McAllister, M., Minekus, M., Muenster, U., Müllertz, A., Verwei, M., Vertzoni, M., Weitschies, W., Augustijns, P., 2014. In vitro models for the prediction of in vivo performance of oral dosage forms. Eur J Pharm Sci 57, 342-366. https://doi.org/10.1016/j.ejps.2013.08.024

Kourentas, A., Vertzoni, M., Stavrinoudakis, N., Symillidis, A., Brouwers, J., Augustijns, P., Reppas, C., Symillides, M., 2016. An in vitro biorelevant gastrointestinal transfer (BioGIT) system for forecasting concentrations in the fasted upper small intestine: Design, implementation, and evaluation. Eur J Pharm Sci 82, 106-114. https://doi.org/10.1016/j.ejps.2015.11.012

Krieg, B.J., Taghavi, S.M., Amidon, G.L., Amidon, G.E., 2015. In Vivo Predictive Dissolution: Comparing the Effect of Bicarbonate and Phosphate Buffer on the Dissolution of Weak Acids and Weak Bases. J Pharm Sci 104, 2894-2904. https://doi.org/10.1002/jps.24460

Krieg, B.J., Taghavi, S.M., Amidon, G.L., Amidon, G.E., 2014. In vivo predictive dissolution: transport analysis of the CO2, bicarbonate in vivo buffer system. J Pharm Sci 103, 3473-3490. https://doi.org/10.1002/jps.24108

Kukura, J., Baxter, J.L., Muzzio, F.J., 2004. Shear distribution and variability in the USP Apparatus 2 under turbulent conditions. Int J Pharm 279, 9-17. https://doi.org/10.1016/j.ijpharm.2004.03.033

Lennernäs, H., Aarons, L., Augustijns, P., Beato, S., Bolger, M., Box, K., Brewster, M., Butler, J., Dressman, J., Holm, R., Julia Frank, K., Kendall, R., Langguth, P., Sydor, J., Lindahl, A., McAllister, M., Muenster, U., Müllertz, A., Ojala, K., Pepin, X., Reppas, C., Rostami-Hodjegan, A., Verwei, M., Weitschies, W., Wilson, C., Karlsson, C., Abrahamsson, B., 2014. Oral biopharmaceutics tools - time for a new initiative - an introduction to the IMI project OrBiTo. Eur J Pharm Sci 57, 292-299. https://doi.org/10.1016/j.ejps.2013.10.012

Lennernäs, H., Lindahl, A., Van Peer, A., Ollier, C., Flanagan, T., Lionberger, R., Nordmark, A., Yamashita, S., Yu, L., Amidon, G.L., Fischer, V., Sjögren, E., Zane, P., McAllister, M., Abrahamsson, B., 2017a. In Vivo Predictive Dissolution (IPD) and Biopharmaceutical Modeling and Simulation: Future Use of Modern Approaches and Methodologies in a Regulatory Context. Mol. Pharm. 14, 1307-1314. https://doi.org/10.1021/acs.molpharmaceut.6b00824 
Lennernäs, H., Lindahl, A., Van Peer, A., Ollier, C., Flanagan, T., Lionberger, R., Nordmark, A., Yamashita, S., Yu, L., Amidon, G.L., Fischer, V., Sjögren, E., Zane, P., McAllister, M., Abrahamsson, B., 2017b. In Vivo Predictive Dissolution (IPD) and Biopharmaceutical Modeling and Simulation: Future Use of Modern Approaches and Methodologies in a Regulatory Context. Mol. Pharm. 14, 1307-1314. https://doi.org/10.1021/acs.molpharmaceut.6b00824

Lionberger, R., Jiang, W., Huang, S.-M., Geba, G., 2013. Confidence in generic drug substitution. Clin. Pharmacol. Ther. 94, 438-440. https://doi.org/10.1038/clpt.2013.104

Matsui, K., Tsume, Y., Amidon, G.E., Amidon, G.L., 2016. The Evaluation of In Vitro Drug Dissolution of Commercially Available Oral Dosage Forms for Itraconazole in Gastrointestinal Simulator With Biorelevant Media. J Pharm Sci 105, 2804-2814. https://doi.org/10.1016/j.xphs.2016.02.020

Matsui, K., Tsume, Y., Amidon, G.E., Amidon, G.L., 2015. In Vitro Dissolution of Fluconazole and Dipyridamole in Gastrointestinal Simulator (GIS), Predicting in Vivo Dissolution and DrugDrug Interaction Caused by Acid-Reducing Agents. Mol. Pharm. 12, 2418-2428. https://doi.org/10.1021/acs.molpharmaceut.5b00135

Matsui, K., Tsume, Y., Takeuchi, S., Searls, A., Amidon, G.L., 2017. Utilization of Gastrointestinal Simulator, an in Vivo Predictive Dissolution Methodology, Coupled with Computational Approach To Forecast Oral Absorption of Dipyridamole. Mol. Pharm. 14, 1181-1189. https://doi.org/10.1021/acs.molpharmaceut.6b01063

Menys, A., Taylor, S.A., Emmanuel, A., Ahmed, A., Plumb, A.A., Odille, F., Alam, A., Halligan, S., Atkinson, D., 2013. Global small bowel motility: assessment with dynamic MR imaging. Radiology 269, 443-450. https://doi.org/10.1148/radiol.13130151

Mudie, D., Brasseur, J.G., Mansfield, J., Wang, Y., Jayaraman, B., Amidon, G.L., 2015. Influence of Fluid Shear Rate on Dissolution Rate of Poorly Soluble Drug Particles; Implications for In vivo Predictive In vitro Dissolution Methodologies and Mechanistic Computational Modeling. Presented at the American Association of Pharmaceutical Scientists, Orlando, FL.

Mudie, D.M., Murray, K., Hoad, C.L., Pritchard, S.E., Garnett, M.C., Amidon, G.L., Gowland, P.A., Spiller, R.C., Amidon, G.E., Marciani, L., 2014. Quantification of gastrointestinal liquid volumes and distribution following a $240 \mathrm{~mL}$ dose of water in the fasted state. Mol. Pharm. 11, 3039-3047. https://doi.org/10.1021/mp500210c

Murray, K., Hoad, C.L., Mudie, D.M., Wright, J., Heissam, K., Abrehart, N., Pritchard, S.E., Al Atwah, S., Gowland, P.A., Garnett, M.C., Amidon, G.E., Spiller, R.C., Amidon, G.L., Marciani, L., 2017. Magnetic Resonance Imaging Quantification of Fasted State Colonic Liquid Pockets in Healthy Humans. Mol. Pharm. 14, 2629-2638. https://doi.org/10.1021/acs.molpharmaceut.7b00095

Pharmaceuticals and Medical Devices Agency, 2012. Guideline for Bioequivalence Studies of Generic Products.

Polli, J.E., 2008. In Vitro Studies are Sometimes Better than Conventional Human Pharmacokinetic In Vivo Studies in Assessing Bioequivalence of Immediate-Release Solid Oral Dosage Forms. AAPS J 10, 289-299. https://doi.org/10.1208/s12248-008-9027-6

Polli, J.E., Abrahamsson, B.S.I., Yu, L.X., Amidon, G.L., Baldoni, J.M., Cook, J.A., Fackler, P., Hartauer, K., Johnston, G., Krill, S.L., Lipper, R.A., Malick, W.A., Shah, V.P., Sun, D., Winkle, H.N., Wu, Y., Zhang, H., 2008. Summary workshop report: bioequivalence, biopharmaceutics classification system, and beyond. AAPS J 10, 373-379. https://doi.org/10.1208/s12248-008-9040-9

Polli, J.E., Yu, L.X., Cook, J.A., Amidon, G.L., Borchardt, R.T., Burnside, B.A., Burton, P.S., Chen, M.-L., Conner, D.P., Faustino, P.J., Hawi, A.A., Hussain, A.S., Joshi, H.N., Kwei, G., Lee, V.H.L., Lesko, L.J., Lipper, R.A., Loper, A.E., Nerurkar, S.G., Polli, J.W., Sanvordeker, D.R., Taneja, R., Uppoor, R.S., Vattikonda, C.S., Wilding, I., Zhang, G., 2004. Summary workshop report: biopharmaceutics classification system--implementation challenges and extension opportunities. J Pharm Sci 93, 1375-1381. https://doi.org/10.1002/jps.20064 
Salehi, N., Hens, B., Job, N., Dean, M., Sinko, P., Amidon, G.E., Ziff, R.M., Amidon, G.L., 2017. Towards a physiologically-relevant in vitro system for oral drug product delivery in the gastrointestinal (GI) tract.

Sinko, P.D., Gidley, D., Vallery, R., Lamoureux, A., Amidon, G.L., Amidon, G.E., 2017. In Vitro Characterization of the Biomimetic Properties of Poly(dimethylsiloxane) To Simulate Oral Drug Absorption. Mol. Pharm. 14, 4661-4674. https://doi.org/10.1021/acs.molpharmaceut.7b00798

Sironi, D., Rosenberg, J., Bauer-Brandl, A., Brandl, M., 2017. Dynamic dissolution-/permeation-testing of nano- and microparticle formulations of fenofibrate. Eur J Pharm Sci 96, 20-27. https://doi.org/10.1016/j.ejps.2016.09.001

Sugihara, M., Takeuchi, S., Sugita, M., Higaki, K., Kataoka, M., Yamashita, S., 2015. Analysis of Intraand Intersubject Variability in Oral Drug Absorption in Human Bioequivalence Studies of 113 Generic Products. Mol. Pharm. 12, 4405-4413. https://doi.org/10.1021/acs.molpharmaceut.5b00602

Talattof, A., Price, J.C., Amidon, G.L., 2016. Gastrointestinal Motility Variation and Implications for Plasma Level Variation: Oral Drug Products. Mol. Pharm. 13, 557-567. https://doi.org/10.1021/acs.molpharmaceut.5b00774

Tsume, Y., Matsui, K., Searls, A.L., Takeuchi, S., Amidon, G.E., Sun, D., Amidon, G.L., 2017. The impact of supersaturation level for oral absorption of BCS class IIb drugs, dipyridamole and ketoconazole, using in vivo predictive dissolution system: Gastrointestinal Simulator (GIS). Eur J Pharm Sci 102, 126-139. https://doi.org/10.1016/j.ejps.2017.02.042

Tsume, Y., Mudie, D.M., Langguth, P., Amidon, G.E., Amidon, G.L., 2014. The Biopharmaceutics Classification System: subclasses for in vivo predictive dissolution (IPD) methodology and IVIVC. Eur J Pharm Sci 57, 152-163. https://doi.org/10.1016/j.ejps.2014.01.009

Tsume, Y., Takeuchi, S., Matsui, K., Amidon, G.E., Amidon, G.L., 2015. In vitro dissolution methodology, mini-Gastrointestinal Simulator (mGIS), predicts better in vivo dissolution of a weak base drug, dasatinib. Eur J Pharm Sci 76, 203-212. https://doi.org/10.1016/j.ejps.2015.05.013

U.S. Government Printing Office, 2003. Code of Federal Regulations Title 21-Food and Drugs. Part 320-Bioavailability and Bioequivalence Requirements. [WWW Document]. URL https://www.gpo.gov/fdsys/pkg/CFR-2003-title21-vol1/content-detail.html (accessed 12.7.17).

Verwei, M., Minekus, M., Zeijdner, E., Schilderink, R., Havenaar, R., 2016. Evaluation of two dynamic in vitro models simulating fasted and fed state conditions in the upper gastrointestinal tract (TIM-1 and tiny-TIM) for investigating the bioaccessibility of pharmaceutical compounds from oral dosage forms. Int J Pharm 498, 178-186. https://doi.org/10.1016/j.ijpharm.2015.11.048

Wakamiya, M., Furukawa, A., Kanasaki, S., Murata, K., 2011. Assessment of small bowel motility function with cine-MRI using balanced steady-state free precession sequence. J Magn Reson Imaging 33, 1235-1240. https://doi.org/10.1002/jmri.22529

Wang, Y., Abrahamsson, B., Lindfors, L., Brasseur, J.G., 2015. Analysis of Diffusion-Controlled Dissolution from Polydisperse Collections of Drug Particles with an Assessed Mathematical Model. J Pharm Sci 104, 2998-3017. https://doi.org/10.1002/jps.24472

Wang, Y., Abrahamsson, B., Lindfors, L., Brasseur, J.G., 2012. Comparison and analysis of theoretical models for diffusion-controlled dissolution. Mol. Pharm. 9, 1052-1066. https://doi.org/10.1021/mp2002818

Wang, Y., Brasseur, J.G., Banco, G.G., Webb, A.G., Ailiani, A.C., Neuberger, T., 2010. A multiscale lattice Boltzmann model of macro- to micro-scale transport, with applications to gut function. Philosophical Transactions of the Royal Society of London A: Mathematical, Physical and Engineering Sciences 368, 2863-2880. https://doi.org/10.1098/rsta.2010.0090

Wright, J., Evans, D., Gowland, P., Mansfield, P., 1999. Validation of antroduodenal motility measurements made by echo-planar magnetic resonance imaging. Neurogastroenterol. Motil. 11, 19-25. 
Yu, A., Baker, J.R., Fioritto, A.F., Wang, Y., Luo, R., Li, S., Wen, B., Bly, M., Tsume, Y., Koenigsknecht, M.J., Zhang, X., Lionberger, R., Amidon, G.L., Hasler, W.L., Sun, D., 2016. Measurement of in vivo Gastrointestinal Release and Dissolution of Three Locally Acting Mesalamine Formulations in Regions of the Human Gastrointestinal Tract. Mol. Pharm. https://doi.org/10.1021/acs.molpharmaceut.6b00641

Yu, A., Jackson, T., Tsume, Y., Koenigsknecht, M., Wysocki, J., Marciani, L., Amidon, G.L., Frances, A., Baker, J.R., Hasler, W., Wen, B., Pai, A., Sun, D., 2017. Mechanistic Fluid Transport Model to Estimate Gastrointestinal Fluid Volume and Its Dynamic Change Over Time. AAPS J. https://doi.org/10.1208/s12248-017-0145-x 